\title{
Real hypersurfaces of complex quadric in terms of star-Ricci tensor
}

\author{
Xiaomin Chen * \\ College of Science, China University of Petroleum (Beijing), \\ Beijing, 102249, China \\ xmchen@cup.edu.cn
}

October 31, 2017

\begin{abstract}
In this article, we introduce the notion of star-Ricci tensors in the real hypersurfaces of complex quadric $Q^{m}$. It is proved that there exist no Hopf hypersurfaces in $Q^{m}, m \geq 3$, with commuting star-Ricci tensor or parallel star-Ricci tensor. As a generalization of star-Einstein metric, star-Ricci solitons on $M$ are considered. In this case we show that $M$ is an open part of a tube around a totally geodesic $\mathbb{C} P^{\frac{m}{2}} \subset Q^{m}, m \geq 4$.
\end{abstract}

Keywords: Hopf hypersurface; complex quadric; commuting star-Ricci tensor; parallel star-Ricci tensor; star-Ricci soliton

$M S C: 53 \mathrm{C} 40,53 \mathrm{C} 15$

\section{Introduction}

The complex quadric $Q^{m}$ is a Hermitian symmetric space $\mathrm{SO}_{m+2} / \mathrm{SO}_{m} \mathrm{SO}_{2}$ with rank two in the class of compact type. It can be regarded as a complex hypersurface of complex projective space $\mathbb{C} P^{m+1}$. Also, the complex quadric $Q^{m}$ can be regarded as a kind of real Grassmannian manifolds of compact type with rank two. In the complex quadric $Q^{m}$ there are two important geometric structures, a complex conjugation structure $A$ and Kähler structure $J$, with each other being anti-commuting, that is, $A J=-J A$. Another distinguish geometric structure in $Q^{m}$ is a parallel rank two vector bundle $\mathfrak{U}$ which contains an $S^{1}$-bundle of real structures, that is, complex conjugations $A$ on the tangent spaces of $Q^{m}$. Here the parallel vector bundle $\mathfrak{U}$ means that $\left(\widetilde{\nabla}_{X} A\right) Y=q(X) A Y$ for all $X, Y \in T_{z} Q^{m}, z \in Q^{m}$, where $\widetilde{\nabla}$ and $q$ denote a connection and a certain 1-form on $T_{z} Q^{m}$, respectively.

*The author is supported by the Science Foundation of China University of PetroleumBeijing(No.2462015YQ0604) and partially supported by the Personnel Training and Academic Development Fund (2462015QZDX02). 
Recall that a nonzero tangent vector $W \in T_{z} Q^{m}, z \in Q^{m}$, is called singular if it is tangent to more than one maximal flat in $Q^{m}$. There are two types of singular tangent vectors for the complex quadric $Q^{m}$ :

1. If there exists a conjugation $A \in \mathfrak{U}$ such that $W \in V(A)$, then $W$ is singular. Such a singular tangent vector is called $\mathfrak{U}$-principal.

2. If there exist a conjugation $A \in \mathfrak{U}$ and orthonormal vectors $X, Y \in V(A)$ such that $W /\|W\|=(X+J Y) / \sqrt{2}$, then $W$ is singular. Such a singular tangent vector is called $\mathfrak{U}$-isotropic.

Let $M$ be a real hypersurface of $Q^{m}$. The Kähler structure $J$ on $Q^{m}$ induces a structure vector field $\xi$ called Reeb vector field on $M$ by $\xi:=-J N$, where $N$ is a local unit normal vector field of $M$ in $Q^{m}$. It is well-known that there is an almost contact structure $(\phi, \eta, \xi, g)$ on $M$ induced from complex quadric. Moreover, if the Reeb vector field $\xi$ is invariant under the shape operator $S$, i.e. $S \xi=\alpha \xi$, where $\alpha=g(S \xi, \xi)$ is a smooth function, then $M$ is said to be a Hopf hypersurface. For the real Hopf hypersurfaces of complex quadric many characterizations were obtained by Suh (see $[9,10,11,12,13]$ etc.). In particular, we note that Suh in [9] introduced parallel Ricci tensor, i.e. $\nabla$ Ric $=0$, for the real hypersurfaces in $Q^{m}$ and gave a complete classification for this case. In addition, if the real hypersurface $M$ admits commuting Ricci tensor, i.e. Ric $\circ \phi=\phi \circ$ Ric, Suh also proved the followings:

Theorem 1.1 ([13]). Let $M$ be a real hypersurface of the complex quadric $Q^{m}, m \geq 3$, with commuting Ricci tensor. Then the unit normal vector field $N$ of $M$ is either $\mathfrak{U}$-principal or $\mathfrak{U}$-isotropic.

Theorem $1.2([13])$. There exist no Hopf real hypersurfaces in the complex quadric $Q^{m}, m \geq 4$, with commuting and parallel Ricci tensor.

Since the Ricci tensor of an Einstein hypersurface in the complex quadric $Q^{m}$ is a constant multiple of $g$, it satisfies naturally commuting and parallelism. Thus we have the following.

Corollary 1.3 ([13]). There exist no Hopf Einstein real hypersurfaces in the complex quadric $Q^{m}, m \geq 4$.

As a generalization of Einstein metrics, recently Suh in [14] has shown a complete classification of Hopf hypersurfaces with a Ricci soliton, which is given by

$$
\frac{1}{2}\left(\mathfrak{L}_{W} g\right)(X, Y)+\operatorname{Ric}(X, Y)=\lambda g(X, Y) .
$$

Here $\lambda$ is a constant and $W$ is a vector field on $M$, which are said to be Ricci soliton constant and potential vector field, respectively, and $\mathfrak{L}_{W}$ denotes the Lie derivative along the direction of the vector field $W$.

Notice that, as the corresponding of Ricci tensor, Tachibana [15] introduced the idea of star-Ricci tensor. These ideas apply to almost contact metric manifolds, and in particular, to real hypersurfaces in complex space forms by Hamada in [3]. The star-Ricci tensor Ric ${ }^{*}$ is defined by

$$
\operatorname{Ric}^{*}(X, Y)=\frac{1}{2} \operatorname{trace}\{\phi \circ R(X, \phi Y)\}, \quad \text { for all } X, Y \in T M .
$$

If the star-Ricci tensor is a constant multiple of $g(X, Y)$ for all $X, Y$ orthogonal to $\xi$, then $M$ is said to be a star-Einstein manifold. Hamada gave a classification of 
star-Einstein hypersurfaces of $\mathbb{C} P^{n}$ and $\mathbb{C} H^{n}$, and further Ivey and Ryan updated and refined the work of Hamada in 2011([4]).

Motivated by the present work, in this paper we introduce the notion of star-Ricci tensor in the real hypersurfaces of complex quadric $Q^{m}$ and study the characterizations of a real Hopf hypersurface whose star-Ricci tensor satifies certain conditions.

First we consider the real hypersurface with commuting star-Ricci tensor, i.e. $\phi \circ$ Ric $^{*}=$ Ric $^{*} \circ \phi$. We assert the following:

Theorem 1.4. There exist no Hopf hypersurfaces of $Q^{m}, m \geq 3$, with commuting star-Ricci tensor.

For the Hopf hypersurfaces of $Q^{m}, m \geq 3$, with parallel star-Ricci tensor, we also prove the following non-existence.

Theorem 1.5. There exist no Hopf hypersurfaces of $Q^{m}, m \geq 3$, with parallel starRicci tensor.

As the generalization of star-Einstein metric Kaimakamis and Panagiotidou [5] introduced a so-called star-Ricci soliton, that is, a Riemannain metric $g$ on $M$ satisfying

$$
\frac{1}{2} \mathcal{L}_{W} g+\operatorname{Ric}^{*}=\lambda g .
$$

In this case we obtain the following characterization:

Theorem 1.6. Let $M$ be a real hypersurface in $Q^{m}, m \geq 4$, admitting a star-Ricci soliton with potential vector field $\xi$, then $M$ is an open part of a tube around a totally geodesic $\mathbb{C} P^{\frac{m}{2}} \subset Q^{m}$.

This paper is organized as follows. In Section 2 and Section 3, some basic concepts and formulas for real hypersurfaces in complex quadric are presented. In Section 4 we consider Hopf hypersurfaces with commuting star-Ricci tensor and give the proof of Theorem 1.4. In Section 5 we will prove Theorem 1.5. At last we assume that a Hopf hypersurface admits star-Ricci soliton and give the proof of Theorem 1.6 as the Section 6 .

\section{The complex quadric}

In this section we will summarize some basic notations and formulas about the complex quadric $Q^{m}$. For more detail see $[1,2,7,6]$. The complex quadric $Q^{m}$ is the hypersurface of complex projective space $\mathbb{C} P^{m+1}$, which is defined by $z_{1}^{2}+\cdots+z_{m+2}^{2}=0$, where $z_{1}, \cdots, z_{m+2}$ are homogeneous coordinates on $\mathbb{C} P^{m+1}$. In the complex quadric it is equipped with a Riemannian metric $\widetilde{g}$ induced from the Fubini-Study metric on $\mathbb{C} P^{m+1}$ with constant holomorphic sectional curvature 4. Also the Kähler structure on $\mathbb{C} P^{m+1}$ induces canonically a Kähler structure $(J, \widetilde{g})$ on the complex quadric $Q^{m}$.

The complex projective space $\mathbb{C} P^{m+1}$ is a Hermitian symmetric space of the special unitary group $\mathrm{SU}_{m+2}$, i.e. $\mathbb{C} P^{m+1}=\mathrm{SU}_{m+2} / S\left(U_{1} U_{m+1}\right)$. The special orthogonal group $\mathrm{SO}_{m+2} \subset \mathrm{SU}_{m+2}$ acts on $\mathbb{C} P^{m+1}$ with cohomogeneity one. The orbit containing $o$ is a totally geodesic real projective space $\mathbb{R} P^{m+1} \subset \mathbb{C} P^{m+1}$, where $o=[0, \cdots, 0,1] \in$ $\mathbb{C} P^{m+1}$ is the fixed point of the action of the stabilizer $S\left(U_{m+1} U_{1}\right)$. We can identify $Q^{m}$ with a homogeneous space $\mathrm{SO}(m+2) / \mathrm{SO}_{2} \mathrm{SO}_{m}$, which is the second singular orbit of this action. Such a homogeneous space model leads to the geometric interpretation of the complex quadric $Q^{m}$ as the Grassmann manifold $G_{2}^{+}\left(\mathbb{R}^{m+2}\right)$ of oriented 2planes in $\mathbb{R}^{m+2}$. From now on we always assume $m \geq 3$ because it is well known that 
$Q^{1}$ is isometric to a sphere $S^{2}$ with constant curvature and $Q^{2}$ is isometric to the Riemannian product of two 2 -spheres with constant curvature.

For a unit normal vector $\rho$ of $Q^{m}$ at a point $z \in Q^{m}$ we denote by $A=A_{\rho}$ the shape operator of $Q^{m}$ in $\mathbb{C} P^{m+1}$ with respect to $\rho$, which is an involution on the tangent space $T_{z} Q^{m}$, and the tangent space can be decomposed as

$$
T_{z} Q^{m}=V\left(A_{\rho}\right) \oplus J V\left(A_{\rho}\right),
$$

where $V\left(A_{\rho}\right)$ is the $(+1)$-eigenspace and $J V\left(A_{\rho}\right)$ is the $(-1)$-eigenspace of $A_{\rho}$. This means that the shape operator $A$ defines a real structure on $T_{z} Q^{m}$, equivalently, $A$ is a complex conjugation. Since the real codimension of $Q^{m}$ in $\mathbb{C} P^{m+1}$ is 2 , this induces an $S^{1}$-subbundle $\mathfrak{U}$ of the endomorphism bundle $\operatorname{End}\left(T Q^{m}\right)$ consisting of complex conjugations. Notice that $J$ and each complex conjugation $A \in \mathfrak{U}$ anti-commute, i.e. $A J=-J A$.

\section{Real hypersurface of complex quadric and its star- Ricci tensor}

Let $M$ be an immersed real hypersurface of $Q^{m}$ with induced metric $g$. There exists a local defined unit normal vector field $N$ on $M$ and we write $\xi:=-J N$ by the structure vector field of $M$. An induced one-form $\eta$ is defined by $\eta(\cdot)=\widetilde{g}(J \cdot, N)$, which is dual to $\xi$. For any vector field $X$ on $M$ the tangent part of $J X$ is denoted by $\phi X=J X-\eta(X) N$. Moreover, the following identities hold:

$$
\begin{aligned}
& \phi^{2}=-I d+\eta \otimes \xi, \quad \eta \circ \phi=0, \quad \phi \circ \xi=0, \quad \eta(\xi)=1, \\
& g(\phi X, \phi Y)=g(X, Y)-\eta(X) \eta(Y), \quad g(X, \xi)=\eta(X),
\end{aligned}
$$

where $X, Y \in \mathfrak{X}(M)$. By these formulas, we know that $(\phi, \eta, \xi, g)$ is an almost contact metric structure on $M$. The tangent bundle $T M$ can be decomposed as $T M=\mathcal{C} \otimes \mathbb{R} \xi$, where $\mathcal{C}=\operatorname{ker} \eta$ is the maximal complex subbundle of $T M$. Denote by $\nabla, S$ the induced Riemannian connection and the shape operator on $M$, respectively. Then the Gauss and Weingarten formulas are given respectively by

$$
\widetilde{\nabla}_{X} Y=\nabla_{X} Y+g(S X, Y) N, \quad \widetilde{\nabla}_{X} N=-S X,
$$

where $\widetilde{\nabla}$ is the connection on $Q^{m}$ with respect to $\widetilde{g}$. Also, we have

$$
\left(\nabla_{X} \phi\right) Y=\eta(Y) S X-g(S X, Y) \xi, \quad \nabla_{X} \xi=\phi S X .
$$

The curvature tensor $R$ and Codazzi equation of $M$ are given respectively as follows(see [9]):

$$
\begin{aligned}
R(X, Y) Z= & g(Y, Z) X-g(X, Z) Y+g(\phi Y, Z) \phi X-g(\phi X, Z) \phi Y-2 g(\phi X, Y) \phi Z \\
& +g(A Y, Z) A X-g(A X, Z) A Y+g(J A Y, Z) J A X-g(J A X, Z) J A Y \\
& +g(S Y, Z) S X-g(S X, Z) S Y \\
g\left(\left(\nabla_{X} S\right) Y-\left(\nabla_{Y} S\right) X, Z\right)= & \eta(X) g(\phi Y, Z)-\eta(Y) g(\phi X, Z)-2 \eta(Z) g(\phi X, Y) \\
& +g(X, A N) g(A Y, Z)-g(Y, A N) g(A X, Z) \\
& +g(X, A \xi) g(J A Y, Z)-g(Y, A \xi) g(J A X, Z)
\end{aligned}
$$

for any vector fields $X, Y, Z$ on $M$. 
At each point $z \in M$ we denote

$$
\mathcal{Q}_{z}=\left\{X \in T_{z} M \mid A X \in T_{z} M \text { for all } A \in \mathfrak{U}_{z}\right\}
$$

by a maximal $\mathfrak{U}$-invariant subspace of $T_{z} M$. For the subspace the following lemma was proved.

Lemma 3.1 (see [10]). For each $z \in M$ we have

- If $N_{z}$ is $\mathfrak{U}$-principal, then $\mathcal{Q}_{z}=\mathcal{C}_{z}$.

- If $N_{z}$ is not $\mathfrak{U}$-principal, there exist a conjugation $A \in \mathfrak{U}$ and orthonormal vectors $X, Y \in V(A)$ such that $N_{z}=\cos (t) X+\sin (t) J Y$ for some $t \in\left(0, \frac{\pi}{4}\right]$. Then we have $\mathcal{Q}_{z}=\mathcal{C}_{z} \ominus \mathbb{C}(J X+Y)$.

For each point $z \in M$ we choose $A \in \mathfrak{U}_{z}$, then there exist two orthonormal vectors $Z_{1}, Z_{2} \in V(A)$ such that

$$
\begin{cases}N & =\cos (t) Z_{1}+\sin (t) J Z_{2} \\ A N & =\cos (t) Z_{1}-\sin (t) J Z_{2} \\ \xi & =\sin (t) Z_{2}-\cos (t) J Z_{1} \\ A \xi & =\sin (t) Z_{2}+\cos (t) J Z_{1}\end{cases}
$$

for $0 \leq t \leq \frac{\pi}{4}$ (see [8, Proposition 3]). From this we get $g(A N, \xi)=0$.

In the real hypersurface $M$ we introduce the star-Ricci tensor Ric* defined by

$$
\operatorname{Ric}^{*}(X, Y)=\frac{1}{2} \operatorname{trace}\{\phi \circ R(X, \phi Y)\}, \quad \text { for all } X, Y \in T M \text {. }
$$

Taking a local frame $\left\{e_{i}\right\}$ of $M$ such that $e_{1}=\xi$ and using (3.4), we derive from (3.7)

$$
\begin{aligned}
& \sum_{i=1}^{2 m-1} g\left(\phi \circ R(X, \phi Y) e_{i}, e_{i}\right) \\
= & g(\phi Y, \phi X)-g\left(X, \phi^{2} Y\right)+g\left(\phi^{2} Y, \phi^{2} X\right)-g\left(\phi X, \phi^{3} Y\right)+2(2 m-2) g(\phi X, \phi Y) \\
& +g(A \phi Y, \phi A X)-g(A X, \phi A \phi Y)+g(J A \phi Y, \phi J A X)-g(J A X, \phi J A \phi Y) \\
& +g(S \phi Y, \phi S X)-g(S X, \phi S \phi Y) \\
= & 4 m g(\phi X, \phi Y)-2 g(A X, \phi A \phi Y)+2 g(J A \phi Y, \phi J A X)-2 g(S X, \phi S \phi Y) .
\end{aligned}
$$

In view of (1.1), the star-Ricci tensor is given by

$$
\begin{aligned}
\operatorname{Ric}^{*}(X, Y)= & 2 m g(\phi X, \phi Y)-g(A X, \phi A \phi Y) \\
& +g(J A \phi Y, \phi J A X)-g(S X, \phi S \phi Y) .
\end{aligned}
$$

Since $A J=-J A$ and $\xi=-J N$, we have

$$
\begin{aligned}
& J A \phi Y=-A J \phi Y=A Y-\eta(Y) A \xi, \\
& \phi J A X=J(J A X)-\eta(J A X) N=-A X+g(N, A X) N .
\end{aligned}
$$

Then

$$
\begin{aligned}
g(J A \phi Y, \phi J A X) & =-g(A X, A Y)+\eta(Y) \eta(X)+g(N, A X) g(A Y, N) \\
& =g\left(\phi^{2} X, Y\right)+g(N, A X) g(A Y, N) .
\end{aligned}
$$


Because

$$
\begin{aligned}
J A \phi Y & =\phi A \phi Y+\eta(A \phi Y) N \\
& =\phi A \phi Y+g(\xi, A J Y-\eta(Y) A N) N \\
& =\phi A \phi Y+g(J \xi, A Y) N \\
& =\phi A \phi Y+g(N, A Y) N,
\end{aligned}
$$

we have

$$
\begin{aligned}
g(A X, \phi A \phi Y) & =g(A X, J A \phi Y-g(N, A Y) N) \\
& =g(A X, J A \phi Y)-g(N, A Y) g(A X, N) \\
& =-g\left(\phi^{2} X, Y\right)-g(N, A Y) g(A X, N) .
\end{aligned}
$$

Thus substituting (3.11) and (3.12) into (3.10) implies

$$
\operatorname{Ric}^{*}(X, Y)=-2(m-1) g\left(\phi^{2} X, Y\right)-2 g(N, A X) g(A Y, N)-g\left((\phi S)^{2} X, Y\right)
$$

for all $X, Y \in T M$.

In the following we always assume that $M$ is a Hopf hypersurface in $Q^{m}$, i.e. $S \xi=\alpha \xi$ for a smooth function $\alpha=g(S \xi, \xi)$. As in [9], since $g(A N, \xi)=0$, by taking $Z=\xi$ in the Codazzi equation (3.8), we have

$$
\begin{aligned}
& g\left(\left(\nabla_{X} S\right) Y-\left(\nabla_{Y} S\right) X, \xi\right) \\
= & -2 g(\phi X, Y)+2 g(X, A N) g(A Y, \xi)-2 g(Y, A N) g(A X, \xi) .
\end{aligned}
$$

On the other hand,

$$
\begin{aligned}
& g\left(\left(\nabla_{X} S\right) Y-\left(\nabla_{Y} S\right) X, \xi\right) \\
= & g\left(\left(\nabla_{X} S\right) \xi, Y\right)-g\left(\left(\nabla_{Y} S\right) \xi, X\right) \\
= & (X \alpha) \eta(Y)-(Y \alpha) \eta(X)+\alpha g((\phi S+S \phi) X, Y)-2 g(S \phi S X, Y) .
\end{aligned}
$$

Comparing the previous two equations and putting $X=\xi$ gives

$$
Y \alpha=(\xi \alpha) \eta(Y)+2 g(Y, A N) g(\xi, A \xi) .
$$

Moreover, we have the following.

Lemma 3.2. ([10, Lemma 4.2]) Let $M$ be a Hopf hypersurface in $Q^{m}$ with (local) unit normal vector field $N$. For each point in $z \in M$ we choose $A \in \mathfrak{U}_{z}$ such that $N_{z}=\cos (t) Z_{1}+\sin (t) J Z_{2}$ holds for some orthonormal vectors $Z_{1}, Z_{2} \in V(A)$ and $0 \leq t \leq \frac{\pi}{4}$. Then

$$
\begin{aligned}
0= & 2 g(S \phi S X, Y)-\alpha g((\phi S+S \phi) X, Y)-2 g(\phi X, Y) \\
& +2 g(X, A N) g(Y, A \xi)-2 g(Y, A N) g(X, A \xi) \\
& +2 g(\xi, A \xi)\{g(Y, A N) \eta(X)-g(X, A N) \eta(Y)\}
\end{aligned}
$$

holds for all vector fields $X, Y$ on $M$.

From this lemma we can prove the following.

Lemma 3.3. Let $M$ be a Hopf hypersurface in complex quadric $Q^{m}$, then

$$
(\phi S)^{2}=(S \phi)^{2} \text {. }
$$


Proof. From the equation (3.15) we assert the followings:

$$
\begin{aligned}
g\left((S \phi)^{2} X, Y\right)= & \frac{1}{2} \alpha g((\phi S+S \phi) \phi X, Y)+g\left(\phi^{2} X, Y\right)-g(\phi X, A N) g(Y, A \xi) \\
& +g(\phi X, A \xi) g(Y, A N)+g(\xi, A \xi) g(\phi X, A N) \eta(Y) \\
g\left((\phi S)^{2} X, Y\right)= & \frac{1}{2} \alpha g(\phi(\phi S+S \phi) X, Y)+g\left(\phi^{2} X, Y\right)-g(X, A N) g(\phi A \xi, Y) \\
& +g(X, A \xi) g(\phi A N, Y)-g(\xi, A \xi) \eta(X) g(\phi A N, Y) .
\end{aligned}
$$

Thus we obtain

$$
\begin{aligned}
g\left((S \phi)^{2} X-(\phi S)^{2} X, Y\right)= & -g(\phi X, A N) g(Y, A \xi)+g(\phi X, A \xi) g(Y, A N) \\
& +g(\xi, A \xi) g(\phi X, A N) \eta(Y)+g(X, A N) g(\phi A \xi, Y) \\
& -g(X, A \xi) g(\phi A N, Y)+g(\xi, A \xi) \eta(X) g(\phi A N, Y) \\
= & \eta(X) g(A N, N) g(Y, A \xi)-g(\xi, A \xi) g(X, A \xi) \eta(Y) \\
& -g(X, A \xi) \eta(Y) g(A N, N)+g(\xi, A \xi) \eta(X) g(Y, A \xi) \\
= & (\eta(X) g(A \xi, Y)-g(X, A \xi) \eta(Y))(g(A N, N)+g(\xi, A \xi)) .
\end{aligned}
$$

Here we have used the following relations:

$$
\begin{aligned}
g(A \xi, \phi X) & =g(A \xi, J X-\eta(X) N)=g(A N, X), \\
g(A \phi X, N) & =g(A J X-\eta(X) A N, N)=-g(X, A \xi)-\eta(X) g(A N, N) .
\end{aligned}
$$

From (3.9), we get $g(A N, N)+g(\xi, A \xi)=0$, which yields (3.16).

\section{Proof of Theorem 1.4}

In this section we suppose that $M$ is a real Hopf hypersurface with commuting star-

Ricci tensor, that is, $\phi \circ \mathrm{Ric}^{*}=\mathrm{Ric}^{*} \circ \phi$. Making use of (3.13), a straightforward computation gives

$$
\begin{aligned}
0= & g\left(\left(\phi \circ \operatorname{Ric}^{*}-\operatorname{Ric}^{*} \circ \phi\right) X, Y\right) \\
= & -\operatorname{Ric}^{*}(X, \phi Y)-\operatorname{Ric}^{*}(\phi X, Y) \\
= & 2 g(N, A X) g(A \phi Y, N)+2 g(N, A \phi X) g(A Y, N) \\
& +g\left(\phi\left[(S \phi)^{2}-(\phi S)^{2}\right] X, Y\right) .
\end{aligned}
$$

Thus Lemma 3.3 implies

$$
g(N, A X) g(A \phi Y, N)+g(N, A \phi X) g(A Y, N)=0 .
$$

Replacing $X$ and $Y$ by $\phi X$ and $\phi Y$ respectively gives

$$
g(N, A \phi X) g(Y, A N)+g(X, A N) g(A \phi Y, N)=0 .
$$

Now, if $X=Y$, we find $g(A N, \phi X) g(A N, X)=0$ for all vector field $X$ on $M$, which means $A N=N$. Therefore we prove the following.

Lemma 4.1. Let $M$ be a Hopf hypersurface of complex quadric $Q^{m}, m \geq 3$, with commuting star-Ricci tensor. Then the unit normal vector field $N$ is $\mathfrak{U}$-principal. 
In terms of (3.17), the star-Ricci tensor (3.13) becomes

$$
\operatorname{Ric}^{*}(X, Y)=(-2 m+1) g\left(\phi^{2} X, Y\right)-\frac{1}{2} \alpha g(\phi(\phi S+S \phi) X, Y) .
$$

Moreover, from (3.15) we obtain

$$
\begin{aligned}
\operatorname{Ric}^{*}(X) & =(-2 m+1) \phi^{2} X-\frac{1}{2} \alpha \phi(\phi S+S \phi) X \\
& =(-2 m+1) \phi^{2} X-\frac{1}{2} \alpha \phi^{2} S X-\frac{1}{4} \alpha^{2}(\phi S+S \phi) X-\frac{1}{2} \alpha \phi X .
\end{aligned}
$$

By virtue of [9, Lemma 4.3] and Lemma 4.1, it implies that $\alpha$ is constant. If $\alpha \neq 0$, making use of the previous formula, we conclude that

$$
0=\phi \operatorname{Ric}^{*}(X)-\operatorname{Ric}^{*}(\phi X)=\frac{1}{2} \alpha(\phi S X-S \phi X)
$$

for all $X \in T M$. That means that the Reeb flow is isometric. In view of [2, Proposition 6.1], the normal vector field $N$ is isotropic everywhere, which is contradictory with Lemma 4.1. Hence $\alpha=0$ and the star-Ricci tensor becomes

$$
\operatorname{Ric}^{*}(X, Y)=(-2 m+1) g\left(\phi^{2} X, Y\right) \text {. }
$$

Now replacing $X$ and $Y$ by $\phi X$ and $\phi Y$ respectively in (3.13) and using (4.20), we get

$$
(2 m-1)(\phi X, \phi Y)=2(m-1) g(X, \phi Y)-2 g(N, A \phi X) g(A \phi Y, N)-g\left((S \phi)^{2} X, Y\right) \text {. }
$$

Interchanging $X$ and $Y$ and applying the resulting equation to subtract the pervious equation, we obtain

$$
g\left((S \phi)^{2} X-(\phi S)^{2} X, Y\right)=4(m-1) g(X, \phi Y)
$$

So from Lemma 3.3, we conclude that

$$
4(m-1) g(X, \phi Y)=0
$$

which is impossible since $m \geq 3$. We finish the proof of Theorem 1.4.

Remark 4.2. Formula (4.20) with $X, Y \in \mathcal{C}$, we have $\operatorname{Ric}^{*}(X, Y)=(2 m-1) g(X, Y)$, namely $M$ is star-Einstein, thus we have proved that there exist no star-Einstein Hopf hyersurfaces in complex quadric $Q^{m}, m \geq 3$, which is analogous to the conclusion of Corallary 1.3 in the introduction.

\section{Proofs of Theorem 1.5}

In this section we assume $M$ is a Hopf hypersurface of $Q^{m}, m \geq 3$, with parallel star-Ricci tensor. In order to prove Theorem 1.5, we first prove the following lemma.

Lemma 5.1. Let $M$ be a Hopf hypersurface of $Q^{m}, m \geq 3$, with parallel star-Ricci tensor. Then the unit normal vector $N$ is either $\mathfrak{U}$-principal or $\mathfrak{U}$-isotropic. 
Proof. Since $\nabla$ Ric $^{*}=0$, differentiating equation (3.13) covariantly along vector field $Z$ gives

$$
\begin{aligned}
0= & 2(m-1) g\left(\left(\nabla_{Z} \phi\right) \phi X+\phi\left(\nabla_{Z} \phi\right) X, Y\right) \\
& +2 g\left(\widetilde{\nabla}_{Z} N, A X\right) g(A Y, N)+2 g\left(N,\left(\widetilde{\nabla}_{Z} A\right) X\right) g(A Y, N) \\
& +2 g\left(\widetilde{\nabla}_{Z} N, A Y\right) g(A X, N)+2 g\left(N,\left(\widetilde{\nabla}_{Z} A\right) Y\right) g(A X, N) \\
& +g\left(\left(\nabla_{Z} \phi\right) S \phi S X, Y\right)+g\left(\phi\left(\nabla_{Z} S\right) \phi S X, Y\right) \\
& +g\left(\phi S\left(\nabla_{Z} \phi\right) S X, Y\right)+g\left(\phi S \phi\left(\nabla_{Z} S\right) X, Y\right) .
\end{aligned}
$$

Here we have used $\left(\widetilde{\nabla}_{Z} A\right) X=q(Z) A X$ for a certain 1-form $q$ as in the introduction. Moreover, by (3.5) we have

$$
\begin{aligned}
0= & -2(m-1) g(S Z, \phi X) \eta(Y)+2(m-1) \eta(X) g(\phi S Z, Y) \\
& -2 g(S Z, A X) g(A Y, N)+4 q(Z) g(N, A X) g(A Y, N) \\
& -2 g(S Z, A Y) g(A X, N)-g(S Z, S \phi S X) \eta(Y)+g\left(\phi\left(\nabla_{Z} S\right) \phi S X, Y\right) \\
& +\eta(S X) g\left(\phi S^{2} Z, Y\right)+g\left(\phi S \phi\left(\nabla_{Z} S\right) X, Y\right) .
\end{aligned}
$$

Since $S \xi=\alpha \xi$, letting $X=\xi$ we get

$$
\begin{aligned}
0= & 2(m-1) g(\phi S Z, Y)-2 g(S Z, A \xi) g(A Y, N) \\
& +\alpha g\left(\phi S^{2} Z, Y\right)+g\left(\left(\nabla_{Z} S\right) \xi, \phi S \phi Y\right) \\
= & 2(m-1) g(\phi S Z, Y)-2 g(S Z, A \xi) g(A Y, N) \\
& +\alpha g\left(\phi S^{2} Z, Y\right)+g(\alpha \phi S Z-S \phi S Z, \phi S \phi Y) .
\end{aligned}
$$

Moreover, if $Z=\xi$ then we get $\alpha g(A \xi, \xi) g(A Y, N)=0$. If $\alpha \neq 0$ then $\cos (2 t) g(A Y, N)=$ 0 by (3.9). That means that $t=\frac{\pi}{4}$ or $A Y \in T M$, that is, the unit normal vector $N$ is $\mathfrak{U}$-principal or $\mathfrak{U}$-isotropic. If $\alpha=0$ then $g(Y, A N) g(\xi, A \xi)=0$ for any $Y \in T M$ by (3.14), thus we have same conclusion. The proof is complete.

We first assume that the unit normal vector field $N$ is $\mathfrak{U}$-isotropic. In this case these expressions in (3.9) become

$$
\begin{cases}N & =\frac{1}{\sqrt{2}}\left(Z_{1}+J Z_{2}\right) \\ A N & =\frac{1}{\sqrt{2}}\left(Z_{1}-J Z_{2}\right) \\ \xi & =\frac{1}{\sqrt{2}}\left(Z_{2}-J Z_{1}\right) \\ A \xi & =\frac{1}{\sqrt{2}}\left(Z_{2}+J Z_{1}\right)\end{cases}
$$

Thus

$$
g(A \xi, \xi)=g(A N, N)=0
$$

So (3.15) becomes

$$
\begin{aligned}
S \phi S X= & \frac{1}{2} \alpha(\phi S+S \phi) X+\phi X \\
& -g(X, A N) A \xi+g(X, A \xi) A N .
\end{aligned}
$$

The formula (5.21) with $Z=\xi$ implies

$$
\begin{aligned}
0= & -2 g(S \xi, A X) g(A Y, N)+4 q(\xi) g(N, A X) g(A Y, N) \\
& -2 g(S \xi, A Y) g(A X, N)-g\left(\left(\nabla_{\xi} S\right) \phi S X, \phi Y\right) \\
& +g\left(\left(\nabla_{\xi} S\right) X, \phi S \phi Y\right) .
\end{aligned}
$$


By Codazzi equation (3.8), we get

$$
\begin{aligned}
\left(\nabla_{\xi} S\right) Y= & \alpha \phi S Y-S \phi S Y+\phi Y-g(Y, A N) A \xi \\
& +g(Y, A \xi) A N \\
= & \frac{1}{2} \alpha(\phi S-S \phi) Y .
\end{aligned}
$$

Thus substituting this into (5.24) gives

$$
\begin{aligned}
0= & -2 \alpha g(\xi, A X) g(A Y, N)+4 q(\xi) g(N, A X) g(A Y, N) \\
& -2 \alpha g(\xi, A Y) g(A X, N)-\frac{1}{2} \alpha g(S \phi S X+\phi S \phi S \phi X, Y) .
\end{aligned}
$$

Moreover, by (5.23) we have $S \phi S X+\phi S \phi S \phi X=0$, thus taking $X=A \xi$ in (5.25) yields

$$
\alpha g(A Y, N)=0
$$

Here we have used $g(A \xi, A \xi)=1$ and $g(A N, A \xi)=0$. From this we derive $\alpha=0$ since $N$ is $\mathfrak{U}$-isotropic.

On the other hand, we put $Y=\xi$ in (5.21) and get

$$
0=2(m-1) g(S Z, \phi X)+2 g(S Z, A \xi) g(A X, N)+g(S Z, S \phi S X) .
$$

Applying (5.23) in the above formula, we have

$$
0=(2 m-1) g(S Z, \phi X)+g(S Z, A \xi) g(A X, N)+g(S Z, A N) g(X, A \xi) .
$$

That is,

$$
0=(2 m-1) S \phi X+g(A X, N) S A \xi+g(X, A \xi) S A N .
$$

When $X=A N$, it comes to

$$
0=(2 m-1) S \phi A N+S A \xi .
$$

Then $A \xi=\phi A N$ implies $S A \xi=0$. Similarly, $S A N=0$. Therefore from (5.26) we obtain $S \phi X=0$ for all $X \in T M$. As $S \xi=0$ we know $S X=0$ for all $X \in T M$, thus $\nabla_{\xi} S=0$, that means that the hypersurface $M$ admits parallel shape operator. But Suh [10] has showed the non-existence of this type hypersurfaces.

In the following if $N$ is $\mathfrak{U}$-principal, that is, $A N=N$, then (3.13) becomes

$$
\operatorname{Ric}^{*}(X, Y)=-2(m-1) g\left(\phi^{2} X, Y\right)-g\left((\phi S)^{2} X, Y\right) .
$$

In this case we see that the star-Ricci tensor is commuting by Lemma 3.3. Thus we see $\alpha=0$ from the proof of Theorem 1.4. In this case, the formulas (5.21) with $Y=\xi$ and (3.15) respectively become $2(m-1) g(S Z, \phi X)+g(S Z, S \phi S X)=0$ and $S \phi S X=\phi X$, respectively. From these two equations we obtain $g(S Z, \phi X)=0$, that is, $\phi S Z=0$. This implies $S Z=\alpha \eta(Z) \xi=0$. As before, this is impossible.

Summing up the above discussion, we complete the proof Theorem 1.5. 


\section{Proof of Theorem 1.6}

In order to prove our theorem, we first give the following property.

Proposition 6.1. Let $M$ be a real hypersurface in $Q^{m}, m \geq 3$, admitting a star-Ricci soliton with potential vector field $\xi$, then $M$ must be Hopf.

Proof. Since $\mathcal{L}_{W} g$ and $g$ are symmetry, the *-Ricci soliton equation (1.2) implies the star-Ricci tensor is also symmetry, i.e. $\operatorname{Ric}^{*}(X, Y)=\operatorname{Ric}^{*}(Y, X)$ for any vector fields $X, Y$ on $M$. It yields from (3.13) that

$$
(\phi S)^{2} X=(S \phi)^{2} X
$$

for all $X \in T M$.

On the other hand, from the star-Ricci soliton equation (1.2) it follows

$$
\operatorname{Ric}^{*}(X, Y)=\lambda g(X, Y)+\frac{1}{2} g((S \phi-\phi S) X, Y) .
$$

By (3.13), we have

$$
\begin{aligned}
& -2(m-1) g\left(\phi^{2} X, Y\right)-2 g(N, A X) g(A Y, N)-g\left((\phi S)^{2} X, Y\right) \\
& =\lambda g(X, Y)+\frac{1}{2} g((S \phi-\phi S) X, Y) .
\end{aligned}
$$

Putting $X=Y=\xi$ gives $\lambda=0$ since $g(A N, \xi)=0$. Therefore the previous formula with $X=\xi$ yields

$$
(\phi S)^{2} \xi=\frac{1}{2} \phi S \xi
$$

Using (6.27) we get $\phi S \xi=0$, which shows $S \xi=\alpha \xi$ with $\alpha=g(S \xi, \xi)$.

Moreover, by (6.28) we have

$$
\operatorname{Ric}^{*}(X)=\frac{1}{2}(S \phi-\phi S) X .
$$

Thus by a straightforward computation we find $\phi \circ \mathrm{Ric}^{*}+\mathrm{Ric}^{*} \circ \phi=0$ since the relation $\phi^{2} S=S \phi^{2}$ holds by Proposition 6.1. Namely the following result holds.

Proposition 6.2. Let $M$ be a real hypersurface in $Q^{m}, m \geq 3$, admitting a star-Ricci soliton with potential vector field $\xi$, then the star-Ricci tensor is anti-commuting.

Next we will compute the convariant derivative of $\phi \circ$ Ric $^{*}+$ Ric $^{*} \circ \phi=0$. First of all, by (6.30) and (3.6), we compute

$$
\begin{aligned}
\left(\nabla_{X} \operatorname{Ric}^{*}\right)(Y)= & \frac{1}{2}\left\{\left(\nabla_{X} S\right) \phi Y+S\left(\nabla_{X} \phi\right) Y-\left(\nabla_{X} \phi\right) S Y-\phi\left(\nabla_{X} S\right) Y\right\} \\
= & \frac{1}{2}\left\{\left(\nabla_{X} S\right) \phi Y+\eta(Y) S^{2} X-\alpha g(S X, Y) \xi\right. \\
& \left.-\alpha \eta(Y) S X+g(S X, S Y) \xi-\phi\left(\nabla_{X} S\right) Y\right\} .
\end{aligned}
$$

Now differentiating $\phi \circ$ Ric $^{*}+\operatorname{Ric}^{*} \circ \phi=0$ convariantly gives

$$
\begin{aligned}
0= & \left(\nabla_{X} \phi\right) \operatorname{Ric}^{*}(Y)+\phi\left(\nabla_{X} \operatorname{Ric}^{*}\right) Y+\left(\nabla_{X} \operatorname{Ric}^{*}\right) \phi Y+\operatorname{Ric}^{*}\left(\nabla_{X} \phi\right) Y \\
= & -g\left(S X, \operatorname{Ric}^{*}(Y)\right) \xi+\phi\left(\nabla_{X} \operatorname{Ric}^{*}\right) Y+\left(\nabla_{X} \operatorname{Ric}^{*}\right) \phi Y+\eta(Y) \operatorname{Ric}^{*}(S X) \\
= & \left.-\frac{1}{2} g(S X, S \phi Y-\phi S Y)\right) \xi+\phi\left(\nabla_{X} \operatorname{Ric}^{*}\right) Y+\left(\nabla_{X} \operatorname{Ric}^{*}\right) \phi Y \\
& +\frac{1}{2} \eta(Y)\left(S \phi S X-\phi S^{2} X\right) .
\end{aligned}
$$


Applying (6.31) in the above formula, we get

$$
\begin{aligned}
0= & g(S X, \phi S Y) \xi+\left\{-\alpha \eta(Y) \phi S X+g\left(\left(\nabla_{X} S\right) Y, \xi\right) \xi\right\} \\
& +\left\{\eta(Y)\left(\nabla_{X} S\right) \xi-\alpha g(S X, \phi Y) \xi\right\}+\eta(Y) S \phi S X \\
= & g(S X, \phi S Y) \xi-\alpha \eta(Y) \phi S X+\{g((Y, X(\alpha) \xi+\alpha \phi S X-S \phi S X)\} \xi \\
& +\eta(Y)\{X(\alpha) \xi+\alpha \phi S X-S \phi S X\}-\alpha g(S X, \phi Y) \xi+\eta(Y) S \phi S X \\
= & 2 g(S X, \phi S Y) \xi+2 \eta(Y) X(\alpha) \xi-2 \alpha g(S X, \phi Y) \xi,
\end{aligned}
$$

i.e.

$$
g(S X, \phi S Y)+\eta(Y) X(\alpha)-\alpha g(S X, \phi Y)=0 .
$$

From this we know $X(\alpha)=0$ by taking $Y=\xi$, i.e. $\alpha$ is constant. Hence formula (6.32) becomes

$$
g(S X, \phi S Y)=\alpha g(S X, \phi Y) .
$$

Now interchanging $X$ and $Y$ and comparing the resulting equation with the previous equation, we have $\alpha(\phi S-\phi S) X=0$, which shows that either $\alpha=0$ or $\phi S=S \phi$. Namely the following lemma has been proved.

Lemma 6.3. Let $M$ be a real hypersurface in $Q^{m}, m \geq 3$, admitting a star-Ricci soliton with potential vector field $\xi$, then either the Reeb flow is isometric, or $\alpha=0$.

If the Reeb flow of $M$ is isometric, Berndt and Suh proved the following conclusion:

Theorem $6.4([2])$. Let $M$ be a real hypersurface of the complex quadric $Q^{m}, m \geq 3$. The Reeb flow on $M$ is isometric if and only if $m$ is even, say $m=2 k$, and $M$ is an open part of a tube around a totally geodesic $\mathbb{C} P^{k} \subset Q^{2 k}$.

In the following we set $\alpha=0$, it follows from (6.32) that

$$
S \phi S X=0, \quad \text { for all } X \in T M .
$$

And it is easy to show that the normal vector $N$ is either $\mathfrak{U}$-principal or $\mathfrak{U}$-isotropic from (3.14). In the following let us consider these two cases.

Case I: $N$ is $\mathfrak{U}$-principal, that is, $A N=N$. We follow from (3.15) that

$$
S \phi S X=\phi X .
$$

By comparing with (6.33) we find $\phi X=0$, which is impossible.

Case II: $N$ is $\mathfrak{U}$-isotropic. Using (6.33), we derive from (3.15)

$$
g(\phi X, Y)=g(X, A N) g(Y, A \xi)-g(Y, A N) g(X, A \xi) .
$$

Using (6.33) again, we learn (6.29) becomes

$$
\begin{aligned}
& -2(m-1) g\left(\phi^{2} X, Y\right)-2 g(N, A X) g(A Y, N) \\
& =\frac{1}{2} g((S \phi-\phi S) X, Y) .
\end{aligned}
$$

Moreover, replacing $Y$ by $\phi Y$ gives

$$
\begin{aligned}
& -2(m-1) g(\phi X, Y)+2 g(N, A X) g(Y, A \xi) \\
& \quad=\frac{1}{2} g((S \phi-\phi S) X, \phi Y) .
\end{aligned}
$$


Here we have used $g(A \phi Y, N)=-g(Y, A \xi)$, which follows from (3.19) and (5.22).

By interchanging $Y$ and $X$ in the formula (6.35) and applying the resulting equation to subtract this equation, we get

$$
\begin{aligned}
& 2 g(N, A X) g(Y, A \xi)-2 g(N, A Y) g(X, A \xi) \\
= & \frac{1}{2} g((S \phi-\phi S) X, \phi Y)+2(m-1) g(\phi X, Y) \\
& -\frac{1}{2} g((S \phi-\phi S) Y, \phi X)-2(m-1) g(\phi Y, X) \\
= & 4(m-1) g(\phi X, Y) .
\end{aligned}
$$

Combining this with (6.34) we get $(m-3) \phi X=0$, which is a contradiction if $m \geq 4$. Hence we complete the proof of Theorem 1.6.

Acknowledgement. The author would like to thank the referee for the valuable comments on this paper.

\section{References}

[1] J. Berndt, Y. J. Suh, Hypersurfaces in Kaehler manifold, Proc. A.M.S. 143 (2015), 2637-2649.

[2] J. Berndt, Y. J. Suh, Real hypersurfaces with isometric Reeb flows in complex quadrics, Inter. J. Math. 24 (2013), 1350050, 18pp.

[3] T. Hamada, Real hypersurfaces of complex space forms in terms of Ricci *-tensor, Tokyo J. Math. 25 (2002), 473-483.

[4] T. A. Ivey, P. J. Ryan, The ${ }^{*}$-Ricci tensor for Hypersurfaces in $\mathbb{C P}^{n}$ and $\mathbb{C H}^{n}$, Tohoku Math. J. 34 (2011), 445-471.

[5] G. Kaimakamis, K. Panagiotidou, *-Ricci solitons of real hypersurfaces in non-flat complex space forms, J. Geom. Phys. 86 (2014), 408-413.

[6] S. Klein, Totally geodesic submanifolds in the complex quadric, Diff. Geom. Appl. 26 (2008) 79-96.

[7] S. Kobayashi, K. Nomizu, Foundations of Differential Geometry, Vol. II, Wiley Classics Library ed., A Wiley-Interscience Publ., 1996.

[8] H. Reckziegel, On the geometry of the complex quadric, in: Geometry and Topology of Submanifolds VIII, Brussels/Nordfjordeid, 1995, World Sci. Publ., River Edge, NJ, 1995, 302-315.

[9] Y. J. SuH, Real hypersurfaces in the complex quadric with parallel Ricci tensor, Adv. Math. 281 (2015), 886-905.

[10] Y. J. Sun, Real hypersurfaces in the complex quadric with Reeb parallel shape operator, Inter. J. Math. 25 (2014) 1450059, 17pp.

[11] Y. J. SuH, Real hypersurfaces in the complex quadric with parallel normal Jacobi operator, Math. Nachr. 289 (2016), 1-10.

[12] Y. J. SuH, Real hypersurfaces in the complex quadric with harmonic curvature, J. Math. Pures. Appl. 106 (2016), 393-410.

[13] Y. J. SuH, Real hypersurfaces in the complex quadric with commuting and parallel Ricci tensor, J. Geom. Phys. 106 (2016), 130-142. 
[14] Y. J. SuH, Pseudo-anti commuting Ricci tensor and Ricci soliton real hypersurfaces in the complex quadric, J. Math. Pure. Appl. 107 (2017), 429-450.

[15] S. TAChibana, On almost-analytic vectors in almost-Kählerian manifolds, Tohoku Math. J. 11 (1959), 247-265. 